\title{
Fungi inhabiting attine ant colonies: reassessment of the genus Escovopsis and description of Luteomyces and Sympodiorosea gens. nov.
}

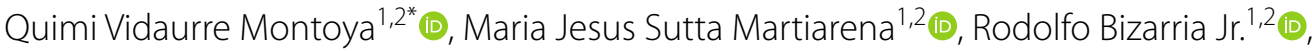

Nicole Marie Gerardo ${ }^{3}$ and Andre Rodrigues ${ }^{1,2^{*}}$

\begin{abstract}
Escovopsis is a diverse group of fungi, which are considered specialized parasites of the fungal cultivars of fungusgrowing ants. The lack of a suitable taxonomic framework and phylogenetic inconsistencies have long hampered Escovopsis research. The aim of this study is to reassess the genus Escovopsis using a taxonomic approach and a comprehensive multilocus phylogenetic analysis, in order to set the basis of the genus systematics and the stage for future Escovopsis research. Our results support the separation of Escovopsis into three distinct genera. In light of this, we redefine Escovopsis as a monophyletic clade whose main feature is to form terminal vesicles on conidiophores. Consequently, E. kreiselii and E. trichodermoides were recombined into two new genera, Sympodiorosea and Luteomyces, as S. kreiselii and L. trichodermoides, respectively. This study expands our understanding of the systematics of Escovopsis and related genera, thereby facilitating future research on the evolutionary history, taxonomic diversity, and ecological roles of these inhabitants of the attine ant colonies.
\end{abstract}

Keywords: Attina, Evolution, Fungus-growing ants, Hypocreaceae, Symbiosis, Systematics, Four new taxa

\section{Introduction}

The kingdom Fungi comprises organisms with wide morphological and genetic diversity (Mueller and Schmit 2007; Hawksworth and Lücking 2017). Through systematic approaches, taxonomists have developed mechanisms to categorize organisms based on their phenotypic and genetic characters (Komarek and Beutel 2006; Pavlinov 2018). Notwithstanding, taxonomic and phylogenetic incongruities like non-monophyly preclude the study of many fungal groups, as is the case for the genus Escovopsis (Ascomycota: Hypocreales, Hypocreaceae).

*Correspondence: quimivimo@gmail.com; andre.rodrigues@unesp.br ${ }^{1}$ Department of General and Applied Biology, São Paulo State University (UNESP), Avenida 24-A, n. 1515, Bela Vista, Rio Claro, SP 13.506-900, Brazil

Full list of author information is available at the end of the article
Escovopsis is a diverse group of fungi, members of which are presumed to have evolved parasitizing the mutualistic fungus of fungus-growing ants (Formicidae: Myrmicinae: Attini: Attina, the "attines") (Yek et al. 2012). Besides, Escovopsis has only been found associated with fungus-growing ant colonies, suggesting that the genus has evolved in relation to these ants' system, potentially for millions of years. Despite its biological importance in relation to a canonical system for the study of coevolution and symbiosis, the paucity of taxonomic studies and unresolved phylogenetic inconsistencies have prevented a comprehensive understanding of the systematics, ecology, and evolution of these hypocrealean fungi.

More than a century has passed since Möller (1893) observed a group of "fungi with strong conidial shapes" (i.e. more prevalent fungi) in attine gardens, which original author(s) and the source, provide a link to the Creative Commons licence, and indicate if changes were made. The images or other third party material in this article are included in the article's Creative Commons licence, unless indicated otherwise in a credit line to the material. If material is not included in the article's Creative Commons licence and your intended use is not permitted by statutory regulation or exceeds the permitted use, you will need to obtain permission directly from the copyright holder. To view a copy of this licence, visit http://creativecommons.org/licenses/by/4.0/. 
80 years later would be named Phialocladus (Kreisel 1972). Almost two decades later, Phialocladus was considered an invalid name because of the lack of the type specimen for its type species, $P$. zsoltii, and consequently, it was renamed as Escovopsis with E. weberi designated as the type species of the latter genus (Muchovej and Della Lucia 1990). Although the macroscopic characters of $E$. weberi were not fully described by the authors, the description of this species established the foundation for Escovopsis taxonomy. In 1995, Seifert et al. (1995) described Escovopsis aspergilloides in a detailed taxonomic study. However, after this study, the taxonomy of Escovopsis was set aside for 18 years.

Unlike systematics studies, the relationship between Escovopsis and the attine ants' mutualistic fungi has been the topic of numerous studies (Currie et al. 2003; Gerardo et al. 2006a, b; Taerum et al. 2007; Folgarait et al. 2011; Elizondo Wallace et al. 2014; Marfetán et al. 2015; Birnbaum and Gerardo 2016; de Man et al. 2016; Heine et al. 2018). A great diversity was attributed to the genus Escovopsis through these studies, yet the morphology and phylogenetic placement of most of the strains named as Escovopsis were not properly assessed. The first ideas about the phylogenetic position of Escovopsis were gradually emerging (Currie et al. 2003; Gerardo et al. 2006b; Augustin et al. 2013; Masiulionis et al. 2015; Meirelles et al. 2015a, b). Initially, some authors suggested that Escovopsis belonged to the Hypocreales, although, no phylogenetic evidence was provided to support that hypothesis at the time (Currie et al. 1999a, b). The first phylogenetic analysis of Escovopsis confirmed the genus to be placed in the Hypocreales, as a sister clade of the Hypocreaceae (Currie et al. 2003). However, a more extensive phylogenetic analysis of Escovopsis strains associated with fungus gardens of Apterostigma ants indicated that the genus belonged to the Hypocreaceae (Gerardo et al. 2006b).

Augustin et al. (2013) were the first authors to combine morphological and phylogenetic approaches to study Escovopsis. Based on internal transcribed spacer (ITS) and large subunit ribosomal RNA (LSU) sequences, the authors described Escovopsis lentecrescens, E. microspora, and E. moelleri, which formed a monophyletic clade with $E$. weberi and E. aspergilloides. The most remarkable character of these newly named species was the presence of conidiophores with vesicles, as previously described by Muchovej and Della Lucia (1990) and Seifert et al. (1995). Nonetheless, while phylogenetic analyses of Augustin et al. (2013) based on ITS and LSU sequences suggested that Escovopsis formed a monophyletic clade, their analyses based on translation elongation factor 1-alpha (tef1) (including all strains treated as Escovopsis at that time except for the ex-type culture of $E$. weberi) suggested that the genus may not be monophyletic.

For almost 30 years, the genus Escovopsis was morphologically defined by the presence of conidiophores with vesicles that support the phialides (i.e., enteroblastic conidiogenous cells), from which conidia are produced. However, that changed with the introduction of Escovopsis trichodermoides (Masiulionis et al. 2015) and E. kreiselii (Meirelles et al. 2015a). These species have conidiophores without vesicles and with poorly differentiated conidiogenous cells (i.e., holoblastic determinate conidiogenous cells with synchronous arrangement; $E$. trichodermoides) and sympodial conidiogenous cells (i.e., holoblastic proliferous conidiogenous cells; E. kreiselii) instead of phialides. Therefore, Meirelles et al. (2015a) amended the morphological description of Escovopsis to insert the morphological features of E. kreiselii. However, because Masiulionis et al. (2015) and Meirelles et al. (2015a) were published at nearly the same time, the new definition did not include E. trichodermoides.

The insertion of E. trichodermoides and E. kreiselii within Escovopsis not only meant changes to the morphological circumscription of the genus but also intensified the phylogenetic uncertainties showed by Augustin et al. (2013). In the phylogenies produced by Masiulionis et al. (2015) and Meirelles et al. (2015a, b), it was clear that vesiculate Escovopsis were more closely related to Escovopsioides (Augustin et al. 2013) than to E. trichodermoides and E. kreiselii. Nonetheless, both Masiulionis et al. (2015) and Meirelles et al. (2015a, b) preferred to maintain E. trichodermoides, E. kreiselii and the vesiculate Escovopsis as placing in the same genus.

Recently, Montoya et al. (2019) used the ITS, LSU, and tef 1 markers in a multilocus phylogenetic approach to describe Escovopsis clavata and E. multiformis. The authors noticed that disagreements in the Escovopsis taxonomy occurred among vesiculate Escovopsis, E. trichodermoides and E. kreiselii. Therefore, they highlighted the need to utilize new molecular markers to resolve the phylogeny of the genus. However, subsequent description of five new Escovopsis species (Marfetán et al. 2018) further complicated Escovopsis taxonomy because interpretation of the phylogenetic analyses made by Marfetán et al. (2018) had some limitations: (1) it was based on the LSU and tef 1 genes separately; (2) the tef 1 sequences obtained in the study do not align with those sequences in previously published studies; (3) some LSU sequences do not have similarity with Escovopsis; and (4) some of the new species (Escovopsis atlas, E. catenulata, and E. pseudoweberi) fall in the same clade, but strains of $E$. atlas fall in different (non-monophyletic) clades.

Given this complicated and piecemeal research history, the aim of this study is to reassess the genus Escovopsis 
by using a comprehensive multilocus phylogeny based on five molecular markers. Our results fill an important gap in mycology and will help future researchers to access the taxonomy, diversity and the evolutionary history of Escovopsis and related genera that inhabit the colonies of fungus-growing ants.

\section{Materials and methods Strains}

A total of 102 strains of Escovopsis, i.e., vesiculate $(\mathrm{n}=64)$ and non-vesiculate $(\mathrm{n}=38)$ species, were included in this study (Additional file 1: Table S1). Of these, 30 strains were obtained from previous studies (Augustin et al. 2013; Masiulionis et al. 2015; Meirelles et al. 2015b; Montoya et al. 2019), and the remaining $(\mathrm{n}=72)$ were isolated from three regions in Brazil (Novo Airão and Camp 41, state of Amazonas; Botucatu, state of São Paulo-Additional file 1: Table S1). The process of isolation, purification, and preservation of the strains followed methods outlined in Montoya et al. (2019). Briefly, from each attine colony, 21 garden fragments $\left(0.5-1 \mathrm{~mm}^{3}\right)$ were inoculated on potato dextrose agar (PDA, Neogen Culture Media, Neogen, Bury) plates (seven fragments per plate) supplemented with chloramphenicol $\left(150 \mu \mathrm{g} \mathrm{mL}^{-1}\right.$, Sigma-Aldrich, St. Louis). The plates were incubated at $25{ }^{\circ} \mathrm{C}$ in darkness and monitored daily for $7 \mathrm{~d}$. When Escovopsis mycelia grown out, they were transferred to new PDA plates without chloramphenicol. Axenic cultures were prepared by single conidial isolation and stored in sterile distilled water kept at $8-10{ }^{\circ} \mathrm{C}$ (Castellani 1963), and in $10 \%$ aqueous solution of glycerol at $-80^{\circ} \mathrm{C}$. Both the strains isolated in this study and those obtained from other studies are deposited at the Laboratory of Fungal Ecology and Systematics (LESF-Department of General and Applied Biology, São Paulo State University (UNESP), Rio Claro, SP, Brazil) and at the UNESPMicrobial Resources Center (CRM-UNESP), Rio Claro, Brazil, under the same conditions.

\section{DNA extraction, PCR and sequencing}

The genomic DNA of the strains was extracted using a modified CTAB method (Möller et al. 1992). Briefly, fungal aerial mycelia, grown for $7 \mathrm{~d}$ at $25^{\circ} \mathrm{C}$ on PDA, were crushed with the aid of glass microspheres (Sigma) in lysis solution and incubated at $65{ }^{\circ} \mathrm{C}$ for $30 \mathrm{~min}$. The organic phase was separated using a solution of chloroform-isoamyl alcohol (24:1). Then, the material was centrifuged $(10,000 \mathrm{~g}$ for $10 \mathrm{~min})$, and the supernatant with the genomic DNA was collected. This extract was precipitated with $3 \mathrm{M}$ sodium acetate and isopropanol and purified with two successive washes of $70 \%$ ethanol. The DNA was suspended in $30 \mu \mathrm{L}$ of Tris-EDTA solution and stored at $-20^{\circ} \mathrm{C}$.
Five molecular markers were amplified for all newly isolated Escovopsis strains: the internal transcribed spacer (ITS), the large subunit ribosomal RNA (LSU), the translation elongation factor 1-alpha (tef1), and the RNA polymerase II protein-coding genes $(r p b 1$ and $r p b 2$, Additional file 1: Table S2). For strains from previous publications, we utilized previously published ITS, LSU and tef 1 sequences, when available, and generated missing sequences for other molecular markers (Additional file 1: Table S1). Sequences of $r p b 1$ and $r p b 2$ for 23 strains in the genus Escovopsioides were also generated in this study to complete our dataset (Additional file 1: Table S1).

PCR reactions were carried out following Meirelles et al. (2015b) for ITS, Meirelles et al. (2015a) for tef 1 and Augustin et al. (2013) for LSU (Additional file 1: Table S2). PCRs for the $r p b 1$ and $r p b 2$ genes (Additional file 1: Table S2) were performed in a final volume of $25 \mu \mathrm{L}(4 \mu \mathrm{L}$ of dNTPs [1.25 mM each]; $5 \mu \mathrm{L}$ of $5 \times$ buffer; $1 \mu \mathrm{L}$ of bovine serum albumin (BSA) [1 mg mL${ }^{-1}$ ]; $2 \mu \mathrm{L}$ of $\mathrm{MgCl}_{2}$ [25 mM]; $1 \mu \mathrm{L}$ of each

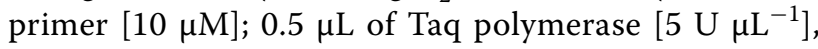
$2 \mu \mathrm{L}$ of diluted genomic DNA [1:100] and $8.5 \mu \mathrm{L}$ of sterile ultrapure water). All PCR reagents were from Promega, Madison. When amplification was difficult, we added $1.5 \mu \mathrm{L}$ of dimethyl sulfoxide (DMSO), decreasing the volume of sterile ultrapure water to $7.0 \mu \mathrm{L}$, or we used the PuReTaq ${ }^{\mathrm{TM}}$ Ready-to-Go ${ }^{\mathrm{TM}}$ PCR kit (illus$\operatorname{tra}^{\mathrm{TM}}$ ) following the manufacturer's protocol. Touchdown PCR conditions were used for $r p b 1$ and $r p b 2$ : (1) $96{ }^{\circ} \mathrm{C}$ for $5 \mathrm{~min}$; (2) 15 cycles of $94{ }^{\circ} \mathrm{C}$ for $30 \mathrm{~s}, 65^{\circ} \mathrm{C}$ for $1.5 \mathrm{~min}$ for $r p b 1$ and for $1 \mathrm{~min}$ for $r p b 2$ (the annealing temperature gradually decreased $1{ }^{\circ} \mathrm{C}$ per cycle) and $72{ }^{\circ} \mathrm{C}$ for $1.5 \mathrm{~min}$ for $r p b 1$ and for $1 \mathrm{~min}$ for $r p b 2$; and then (3) 35 cycles of $94{ }^{\circ} \mathrm{C}$ for $30 \mathrm{~s}, 50{ }^{\circ} \mathrm{C}$ for $1 \mathrm{~min}$ and $72{ }^{\circ} \mathrm{C}$ for $1 \mathrm{~min}$ (Additional file 1: Table S2).

Final amplicons were purified with the Wizard SV Gel and PCR Clean-up System (Promega, Madison) following the manufacturer's protocol. Sequences (forward and reverse) were generated on an ABI3500 (ThermoFisher Scientific, Waltham), and the consensus sequences were assembled in BioEdit v. 7.1.3 (Hall 1999) or Geneious (Kearse et al. 2012). All sequences are deposited in GenBank (Additional file 1: Table S1 for accession numbers).

\section{Phylogenetic analyses}

In order to have a complete perspective of the Escovopsis' phylogenetic incongruences and their possible solutions, we performed phylogenetic analysis to: (1) know the phylogenetic placement of all strains currently treated as Escovopsis, and (2) provide a phylogenetic framework that establishes the foundations of the genus' systematics. 


\section{Phylogenetic placement of fungi treated as Escovopsis}

We reconstructed a phylogenetic tree combining all tef 1 sequences of fungi treated as Escovopsis in the literature (Currie et al. 2003; Gerardo et al. 2004, 2006b; Taerum et al. 2007, 2010; Augustin et al. 2013; Masiulionis et al. 2015; Meirelles et al. 2015a, b; Montoya et al. 2019) with our tef 1 data set and the tef 1 sequences from other genera of the Hypocreales (Additional file 1: Table S3). It was not possible to perform a multilocus analysis because few strains on the literature were sequenced for more than one molecular marker. Nonetheless, the tef 1 gene was the one used in most of the studies published already. The final data set contained a total of 440 tef 1 sequences (754 bp), that included vesiculate-Escovopsis $(\mathrm{n}=274$ strains), non-vesiculate Escovopsis ( $\mathrm{n}=105$ strains), 60 strains from five Hypocreaceae genera, i.e., Escovopsioides, Hypomyces (along with species under its anamorphic genus Cladobotryum) (Põldmaa 2011), Protocrea, Sphaerostilbella, and Trichoderma, and Lecanicillium antillanum CBS 350.85 as the outgroup (Additional file 1: Table S3). The tef1 sequences of species described in Marfetán et al. (2018) were not included in this analysis because they do not align with the Escovopsis sequences from the previous studies.

The data set was first aligned in MAFFT v.7 (Katoh and Standley 2013), and phylogenetic trees were reconstructed using maximum likelihood (ML) and Bayesian inferences (BI) in RAxML (Stamatakis 2014) and MrBayes v.3.2.2 (Ronquist et al. 2012), respectively. The nucleotide substitution model was GTR for ML and $\mathrm{K} 80+\mathrm{G}$ for BI and was calculated in jModelTest 2 (Darriba et al. 2012), using the Akaike Information Criterion (AIC) with 95\% confidence intervals. For ML analysis, 1000 independent trees and 1000 bootstrap replicates were performed, while for BI two separate runs (each consisting of three hot chains and one cold chain) were carried out. In the last case, five million generations of the Markov Chain Monte Carlo (MCMC) were enough to reach convergence (standard deviation (SD) of split frequencies fell below 0.01). To generate final BI tree, the first $25 \%$ of trees were discarded as burn-in. The final tree was edited in FigTree v.1.4 (http://tree.bio.ed.ac.uk/softw are/figtree/) and Adobe Illustrator CC v.17.1.

\section{Phylogenetic framework for Escovopsis' systematics}

Phylogenetic analyses were performed at order and family levels. The objective of the analyses at the order level was to investigate whether all clades formally described as Escovopsis belong to the Hypocreaceae close to the Cordycipitaceae as previously observed by Augustin et al. (2013). The objective of the analyses at the family level was to investigate whether the vesiculate species of Escovopsis form a monophyletic clade, separating them from the non-vesiculate species, as previously observed by Montoya et al. (2019). Besides, we wanted to know if the monophyly of all those clades remains constant considering the Genealogical Concordance Phylogenetic Species Recognition (GCPSR) concept (Taylor et al. 2000 ), although it was applied to the consideration on the generic differentiation.

Escovopsis species described by Marfetán et al. (2018) were not included in this analyses because: (1) the inavailability of ITS, $r p b 1$, and $r p b 2$ sequences, (2) the tef 1 sequences provided by the authors do not align with the Escovopsis sequences from other studies (Augustin et al. 2013; Masiulionis et al. 2015; Meirelles et al. 2015a, b; Montoya et al. 2019), and (3) the LSU sequences from $E$. longivesica, provided by the authors, do not have similarity with Escovopsis but with Ceriporia alachuana (95.4\% identity, E. longivesica E5, E9) and Penicillium glabrum (95.3\% identity, E. longivesica E10). However, the remaining LSU sequences, identical to Escovopsis, generated by the authors were combined with the LSU data to show its phylogenetic placement (Additional file 1: Table S4 and Additional file 2: Fig. S1).

For the 'order-level', we used a concatenated data set that included 145 sequences for LSU (625 bp), 143 for $r p b 1$ (851 bp), 143 for $r p b 2$ (980 bp), and 144 for tef1 (849 bp) from six families of the order Hypocreales (Bionectriaceae, Clavicipitaceae, Cordycipitaceae, Hypocreaceae, Nectriaceae, and Ophiocordycipitaceae, Additional file 1: Table S5). For this analysis, we generated the $r p b 1$ and $r p b 2$ sequences for Escovopsis (including the nine ex-type strains) and Escovopsioides nivea (including the ex-type strain). All other sequences were obtained from the NCBI GenBank database (Currie et al. 2003; Sung et al. 2008; Augustin et al. 2013; Masiulionis et al. 2015; Meirelles et al. 2015a, b; Montoya et al. 2019, Additional file 1: Table S5). The Stachybotrys clade was used to root the tree (Sung et al. 2008).

Multiple loci were used to address the 'family-level' questions, and all possible combinations of ITS, LSU, tef1, and $r p b 2$ (25 combinations) were analyzed (GCPSR concept; Taylor et al. 2000). Data sets included 133 sequences of ITS (707 bp), LSU (594 bp), tef1 (758 bp), and $r p b 2$ (1023 bp); and 131 sequences of $r p b 1$ (725 bp). The sequences represented 102 strains from the Escovopsis clade (vesiculate $(\mathrm{n}=64)$ and non-vesiculate $(\mathrm{n}=38)$ species, including the nine ex-type strains), 30 strains from five Hypocreaceae genera (Escovopsioides, Hypomyces along with species under its anamorphic genus Cladobotryum, Protocrea, Sphaerostilbella, and Trichoderma), and Lecanicillium antillanum CBS 350.85 as the outgroup (Additional file 1: Table S1).

For all analyses, datasets were first aligned separately for each gene in MAFFT v.7 (Katoh and Standley 2013). 
The nucleotide substitution model for each alignment was calculated in jModelTest 2 (Darriba et al. 2012), using the Akaike Information Criterion (AIC) with 95\% confidence intervals. Then, the datasets were concatenated in Winclada v.1.00.08 (Nixon 2002). All phylogenetic trees were constructed using ML in RAxML v.8 (Stamatakis 2014) and BI in MrBayes v.3.2.2 (Ronquist et al. 2012). For ML, we estimated 1000 independent trees and performed 1000 bootstrap replicates using the GTR + I + G model for each partition independently. For BI analyses, we carried out two separate runs (each consisting of three hot chains and one cold chain) using the GTR + I + G model for each partition independently; for all analyses, two million generations of the Markov Chain Monte Carlo (MCMC) were enough to reach convergence [standard deviation (SD) of split frequencies fell below 0.01]. To generate BI trees, the first $25 \%$ of trees were discarded as burn-in. Trees were edited in FigTree v.1.4 (http://tree.bio.ed.ac.uk/software/figtree/) and Adobe Illustrator CC v.17.1.

\section{Morphology}

We examined the microscopic structures of nine ex-type cultures of Escovopsis species (E. aspergilloides, E. clavata, E. kreiselii, E. lentecrescens, E. microspora, E. moelleri, E. multiformis, E. trichodermoides, and E. weberi), representing the known diversity of the genus, to determine if those morphological features support the results observed in phylogenetic analyses.

To assess and compare the microscopic structures (i.e., conidiophores, conidiophore branches, vesicles, conidiogenous cells, conidia, and chlamydospores) and their features (i.e., shape and pattern), we carried out slide culture preparations on PDA. To do so, we placed three cylinders of PDA (ca. $5 \mathrm{~mm}$ in diameter $\times 5 \mathrm{~mm}$ in height) on a sterilised microscopic slide, and we then inoculated each fragment with conidia of the fungus. Each inoculated fragment was covered with a coverslip and incubated at $25^{\circ} \mathrm{C}$ for $4-7 \mathrm{~d}$ in the dark. After that, the fragments of PDA were removed and the coverslips with fungal mycelia were placed on slides with a drop of lactophenol. The slides were examined under a light microscope (DM750, Leica, Wetzlar), and the microscopic fungal structures were photographed using the software LAS EZ v.4.0 (Leica, Wetzlar).

\section{Results}

\section{Phylogenetic placement of fungi previously known as Escovopsis}

The tef 1 phylogenetic tree showed nine well supported monophyletic clades within the Hypocreaceae (Fig. 1 and Additional file 3: Fig. S2). The four previously recognized genera of the Hypocreaceae, i.e., Escovopsioides,
Hypomyces (along with species under its anamorphic genus Cladobotryum), Protocrea, and Trichoderma, preserved their monophyly. On the other hand, the genus Escovopsis (clades A-E) was found polyphyletic and scattered throughout the family (Fig. 1 and Additional file 3: Fig. S2).

While clades A-E are treated as Escovopsis, clades A (vesiculate Escovopsis; the type species of Escovopsis, i.e., E. weberi belongs to this clade), B (E. trichodermoides), and $\mathrm{E}$ (E. kreiselii) are the only clades containing formally described Escovopsis species, so far. Although clades A and B are sister clades, they are far away separated from the clade $\mathrm{E}$ (paraphyletic clade; Fig. 1 and Additional file 3: Fig. S2). On the other hand, clades C and D introduced by Gerardo et al. (2006b) as yellow and white Escovopsis, respectively (Fig. 1 and Additional file 3: Fig. S2), are more closely related to Escovopsioides and Hypomyces than to the clade A of Escovopsis (paraphyletic).

\section{Phylogenetic framework to redefine the genus Escovopsis}

Phylogenetic analyses (BI and ML) at the order level placed both the vesiculate and non-vesiculate Escovopsis within the Hypocreaceae (Fig. 2). Vesiculate Escovopsis, i.e., E. aspergilloides, E. clavata, E. lentecrescens, E. microspora, E. moelleri, E. multiformis and E. weberi, formed a monophyletic clade in both the BI and ML analyses (BI Posterior Probability (PP) $=1$; ML bootstrap value $(\mathrm{MLB})=100)($ Fig. 2). They can be called as the true Escovopsis. On the other hand, non-vesiculate Escovopsis, i.e., E. kreiselii and E. trichodermoides, formed well-supported, monophyletic clades $(\mathrm{PP}=1, \mathrm{MLB}=100$, each one) respectively outside of the vesiculate Escovopsis and are separated from all other Hypocreaceae genera (Fig. 2).

Analyses on the family level based on GCPSR (Taylor et al. 2000) revealed that Hypomyces, Escovopsioides, E. kreiselii, E. trichodermoides, and vesiculate-Escovopsis each form separate, monophyletic clades (Fig. 3). The phylogenetic placement of the five clades varies depending on the molecular marker used for the analysis. The analyses made separately with ITS, LSU, and $r p b 1$ (Fig. 3A, B, D, respectively), as well as the concatenated analysis based on the five markers (Fig. 3F) indicate vesiculate-Escovopsis, and E. kreiselii as sister clades. The analyses made separately with tef1 and $r p b 2$ (Fig. 3C, $\mathrm{E}$, respectively), and the concatenated analysis based on four markers (LSU, tef1, rpb1, and $r p b 2$-Fig. 3G) indicate vesiculate-Escovopsis and E. trichodermoides as forming sister clades. In addition, the analyses performed with ITS and the five concatenated-markers showed Escovopsioides and E. trichodermoides forming a monophyletic clade $(\mathrm{PP}=1, \mathrm{MLB}=98-$ Fig. $3 \mathrm{~A}$ and $\mathrm{PP}=1$, MLB $<70-$ Fig. 3F, respectively). The analysis based on 


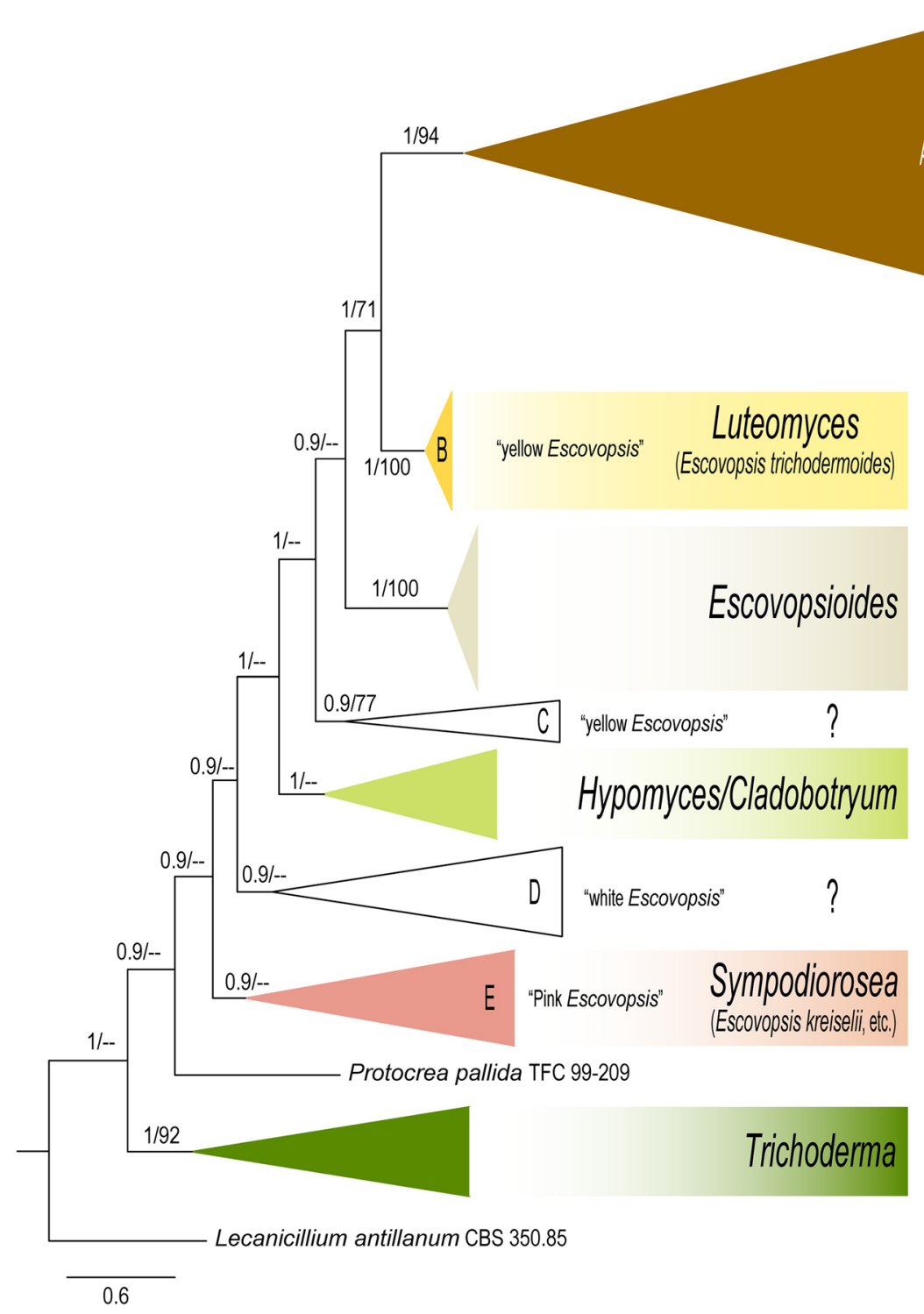

Fig. 1 Collapsed phylogenetic tree (from Additional file 3: Fig. S2) indicating the placement of every isolate previously treated as Escovopsis. The tree shown was inferred using Bayesian Inference (BI). The tree gathers all available tef1 sequences found in the literature with the data set used in this study, including the sequences of the nine Escovopsis ex-type cultures. The tree contains a total of 440 sequences which include: 274 strains of vesiculate-Escovopsis (Clade A), 105 strains of non-vesiculate Escovopsis (Clades B, C, D, E) and 60 strains from four genera, i.e., Escovopsioides, Hypomyces (along with species under its anamorphic genus Cladobotryum), Protocrea, and Trichoderma in the Hypocreaceae. Lecanicillium antillanum CBS 350.85 is used as the outgroup of the tree (see Additional file 1:Table S3 for all strains and their associated metadata used to infer this phylogenetic tree). There is only information, in the literature, on the colour of the colonies of the clades $C$ and $D$, but the microscopic features of these clades are unknown. Numbers on branches indicate BI posterior probabilities (PP) and Maximum Likelihood bootstrap support values (MLB), respectively. Hyphens (--) indicate $M L B<70 \%$

rpb1, however, indicated Escovopsioides forming a monophyletic clade with Hypomyces (Fig. 3D), while analysis based on tef1 placed Hypomyces between Escovopsioides and E. kreiselii (Fig. 3C). Finally, the analyses carried out with LSU, tef1, and rpb2, separately (Fig. 3B, C, E, respectively), and the concatenated analysis (LSU, tef1, rpb1 and rpb2-Fig. 3G) placed Hypomyces, Escovopsioides, E. kreiselii, E. trichodermoides, and vesiculate-Escovopsis in well-supported, monophyletic clades (clearly separated from one another). It is important to highlight that the analysis at this level also showed that $E$. aspergilloides, $E$. clavata, E. lentecrescens, E. moelleri, and E. multiformis and four unnamed phylogenetic species, i.e., LESF 325, LESF 962, and strain groups (LESF 052, LESF 975 and 
(See figure on next page.)

Fig. 2 Phylogenetic tree indicating the placement of Escovopsis (brown branches), Sympodiorosea (pink branches) and Luteomyces (yellow branches) within the Hypocreales. The tree shown was inferred using Bayesian Inference (BI). Highlighted clades in different colours represent six different families (Bionectriaceae, Clavicipitaceae, Cordycipitaceae, Hypocreaceae, Nectriaceae, and Ophiocordycipitaceae) in the Hypocreales. The analysis was based on concatenated sequences of LSU, tef1, rpb1 and rpb2 (See Additional file 1: Table S5 for all strains and their associated metadata used to infer this phylogenetic tree). Numbers on branches indicate Bl posterior probabilities (PP) and Maximum Likelihood bootstrap support values (MLB), respectively. Hyphens (--) indicate MLB $<70 \%$. We used eight species from the family Stachybotryaceae (dark green box) to root the tree. ET indicates ex-type cultures

LESF 979) and (LESF 969, LESF 997, LESF 1003 and LESF 996) formed well-supported monophyletic clades, that were clearly separated from one another (Fig. 3G). Nonetheless, the ex-type cultures of $E$. weberi and $E$. microspora grouped together with 45 other isolates in the same well-supported clade (Fig. 3G).

Regarding the five species described by Marfetán et al. (2018), our analysis of the available LSU data indicates that they form two different clades closely related to $E$. aspergilloides and E. lentecrescens (Additional file 2: Fig. $\mathrm{S} 1)$. Specifically, the four strains of $E$. primorosea formed a well-supported monophyletic clade (Additional file 2: Fig. S1), however E. atlas, E. catenulata, E. pseudoweberi, and five strains named as $E$. weberi by the authors formed a single monophyletic clade (Additional file 2: Fig. S1). This result apparently does not support the new species hypothesis. Future research will be required to clarify the existence of these species.

\section{Morphological evidence to redefine the genus Escovopsis}

Based on morphological analysis, three different groups of fungi are clearly distinguished in the genus Escovopsis. They are: (1) the vesiculate group, composed of $E$. aspergilloides, E. clavata, E. lentecrescens, E. microspora, E. moelleri, E. multiformis, E. weberi, together with the four unnamed phylogenetic species, i.e., LESF 325, LESF 962 and strain groups A (LESF 052, LESF 975 and LESF 979) and B (LESF 969, LESF 996, LESF 997, and LESF 1003); (2) the non-vesiculate group 1, including E. kreiselii and the three unnamed phylogenetic species, i.e., LESF 889, LESF 1010 and the strain group (LESF 864, LESF 886 and LESF 887); and (3) the non-vesiculate group 2 as $E$. trichodermoides.

As the first group, vesiculate Escovopsis spp. present conidiophores with one apical vesicle (mono-vesiculate) or with two to more vesicles (poly-vesiculate) (Fig. 4). Mono-vesiculate conidiophores (Fig. 4A, B) emerge from aerial mycelia in an alternating and opposite pattern. Poly-vesiculate conidiophores (Fig. 4C-H) also emerge from the aerial mycelia and can present short branches composed of one or two cells or long branches with multiple cells. Some poly-vesiculate conidiophores have a swollen cell at the apex from where branches emerge (Fig. 4F). Conidiophores that have swollen cells form their branches only from swollen cells. Vesicles, mostly composed of a single cell (non-septate vesicles-Fig. 4I$\mathrm{T}$ ), and rarely of two cells (septate vesicles-Fig. 4U, V). Vesicles can emerge from the apices and axes of the conidiophore and its branches (usually only one vesicle in the short branches and two or more in long branches) (Fig. 4G, H). Vesicles are connected to the hypha from where they emerge by a basal septum or by a peduncle made up of one or two cells. Vesicles can exhibit different shapes (globose, subglobose, capitate, obovoid, prolate, spatulate, clavate, cymbiform, lanceolate, subulate, cylindric, filiform, clavate-septate, cylindric-septate) (Fig. 4I$\mathrm{V})$ and sizes, depending on the species. Phialides (i.e., enteroblastic conidiogenous cells; Fig. 4W-Z) emerge mainly from the vesicles and less frequently from the aerial mycelia. These structures are lageniform and ampulliform, forming conidia. Main differences of phialides among the different species are mostly related to the sizes of their base, the widened part and the neck (Fig. 4W-Z). Conidia are single-celled, produced in chains from the phialides (enteroblastic conidiogenesis), and can vary in shape (globose, subglobose, ellipsoidal, oblong, and oblong-ornamented) (Fig. 4a-e). Conidia can be smooth or with ornamentations on cell walls (Fig. 4e). Chlamydospores are rarely observed.

The two non-vesiculate groups form conidiophores remarkably different from those of the vesiculate group. Conidiophores of E. trichodermoides lack vesicles and are mostly pyramidal (Trichoderma-like), with one to six short levels of branches arising at more or less right angles from the conidiophore axis (Fig. 5A, B). In contrast to vesiculate Escovopsis, this species has poorly differentiated conidiogenous cell (i.e., holoblastic determinate conidiogenous cells with synchronous arrangement; Fig. 5C-F) with ampulliform shapes producing either solitary (Fig. 5C) or up to three conidia (Fig. 5D-F). The conidia are subglobose to obovate, yellow-brown, mostly ornamented (verrucose; Fig. 5G) and rarely smooth (Fig. $5 \mathrm{H})$. Unlike the vesiculate Escovopsis spp., E. trichodermoides regularly forms chlamydospores (Fig. 5I).

Conidiophores of E. kreiselii also lack vesicles and are formed on the aerial mycelium in an alternated or opposite pattern (Fig. 6A, B), and are more branched (with 


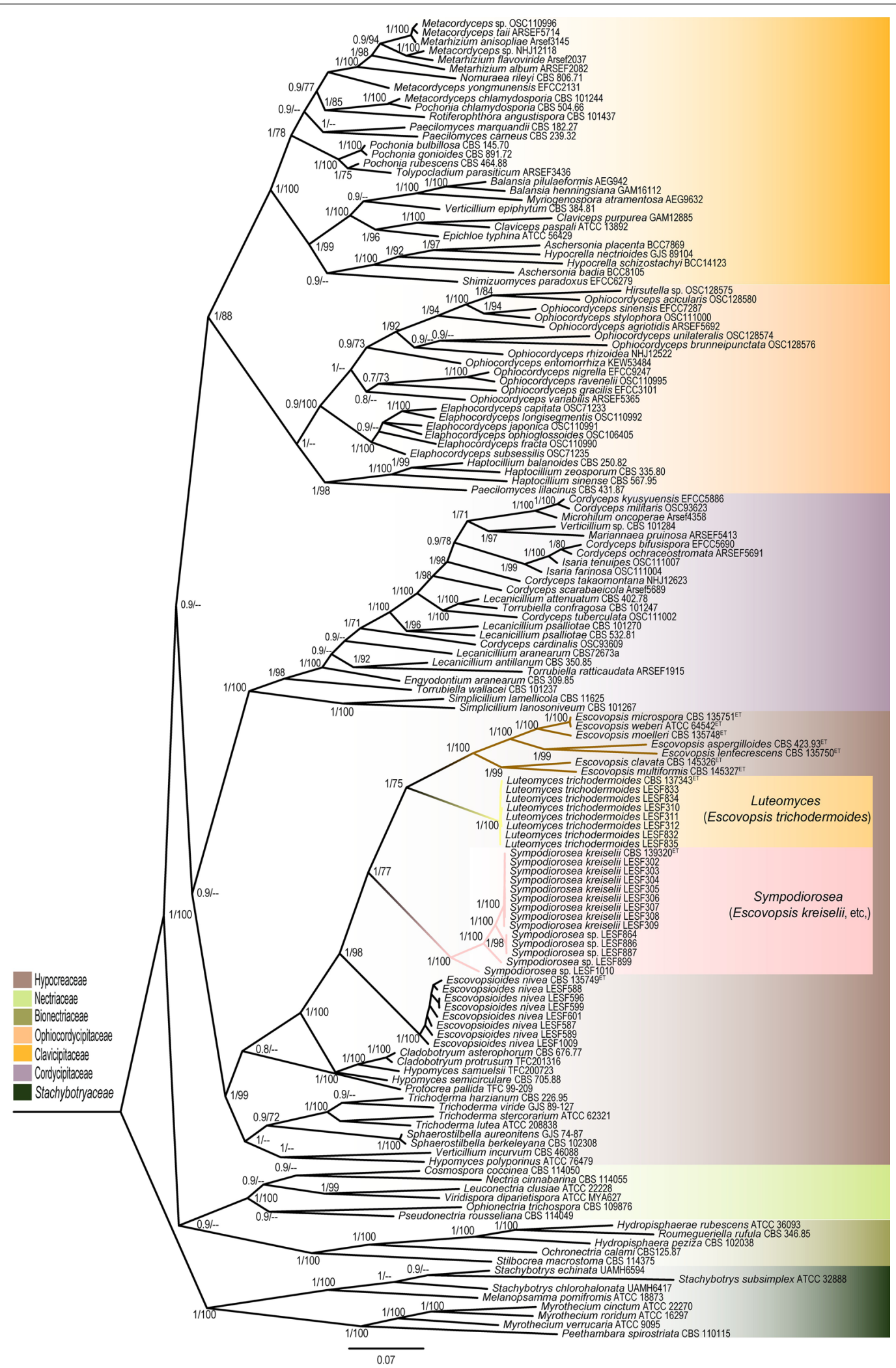

Fig. 2 (See legend on previous page.) 


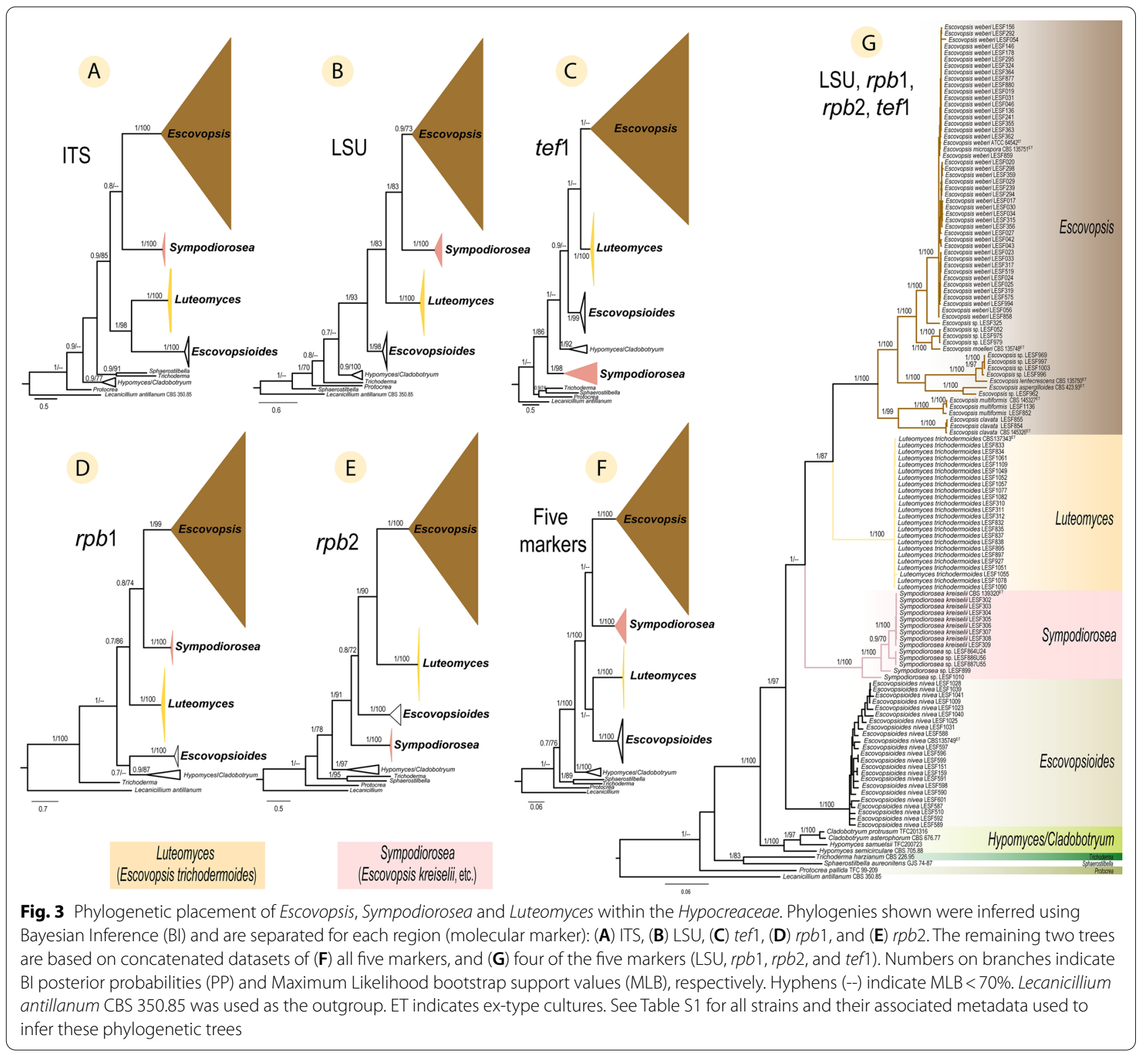

irregular branching conformation) than those of vesiculate Escovopsis. Conidiophores are usually irregularly shaped but some can be pyramidal (Trichoderma-like). Conidiogenous cells of E. kreiselii are not phialidic but sympodial (i.e., holoblastic proliferous conidiogenous cells; Fig. $6 \mathrm{C}-\mathrm{H}$ ), and they are formed on the apex and on both the main and branch axes of the conidiophore (Fig. 6B). Conidia of E. kreiselii are solitary, globose to subglobose, smooth but with thick-walled, light brown to dark brown, and usually with a denticle (Fig. 6I) or with a lesion (when the denticle remains on the conidiogenous cell; Fig. 6J). Like E. trichodermoides, E. kreiselii also forms chlamydospores regularly (Fig. 6K).

\section{Taxonomy}

Phylogenetic analyses in this study unambiguously demonstrate that species previously treated as Escovopsis form three distinct monophyletic clades (Fig. 3). These groups also differ significantly from one another in morphological characters. The first clade (Fig. 3G) includes the ex-type cultures of $E$. weberi (the type species of Escovopsis), E. aspergilloides, E. clavata, E. lentecrescens, E. microspora, E. moelleri, E. multiformis, and four undescribed phylogenetic species, i.e., two strains (LESF 325 and LESF 962) and two groups of strains (LESF 052, LESF 


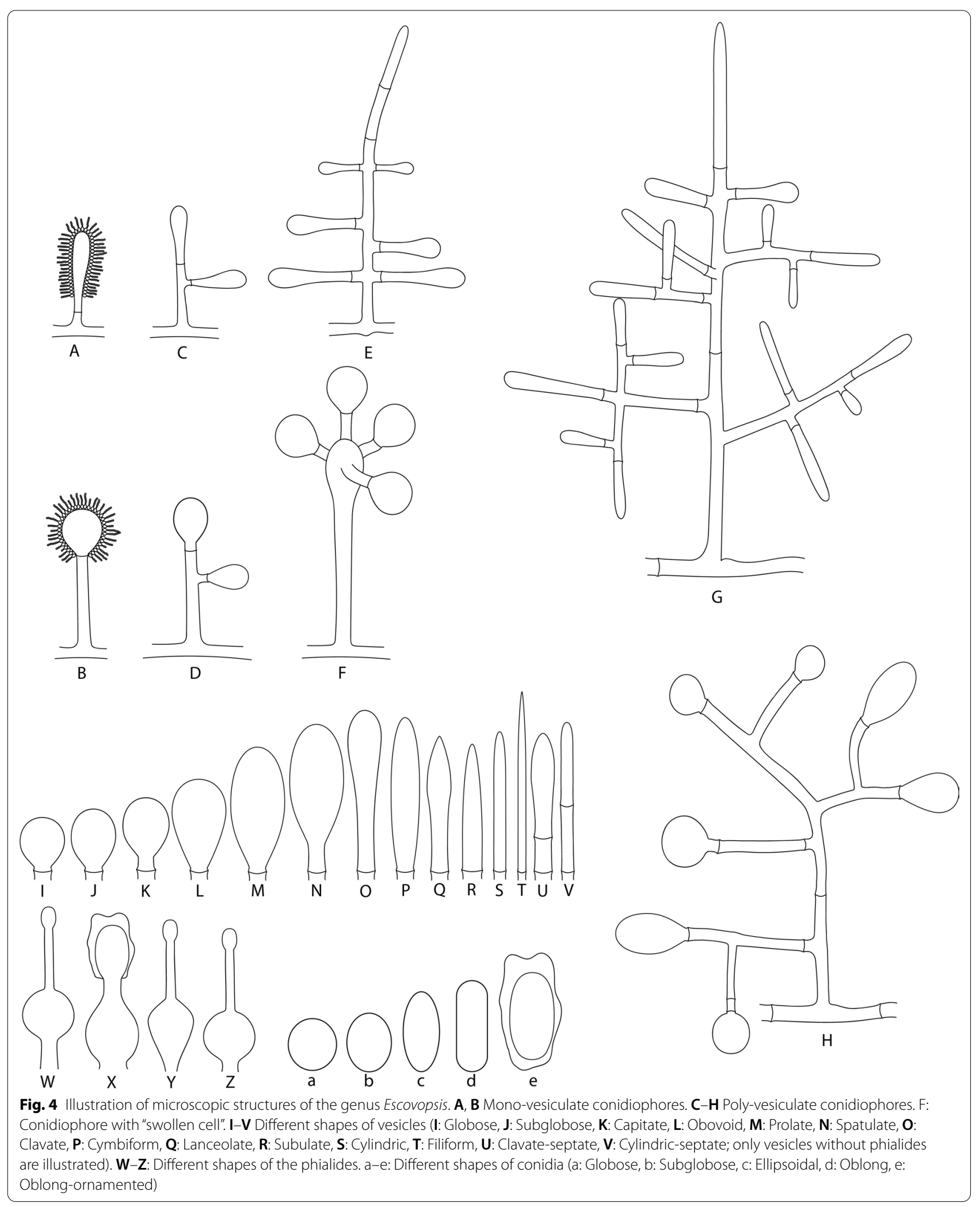


Montoya et al. IMA Fungus

(2021) 12:23

Page 11 of 18

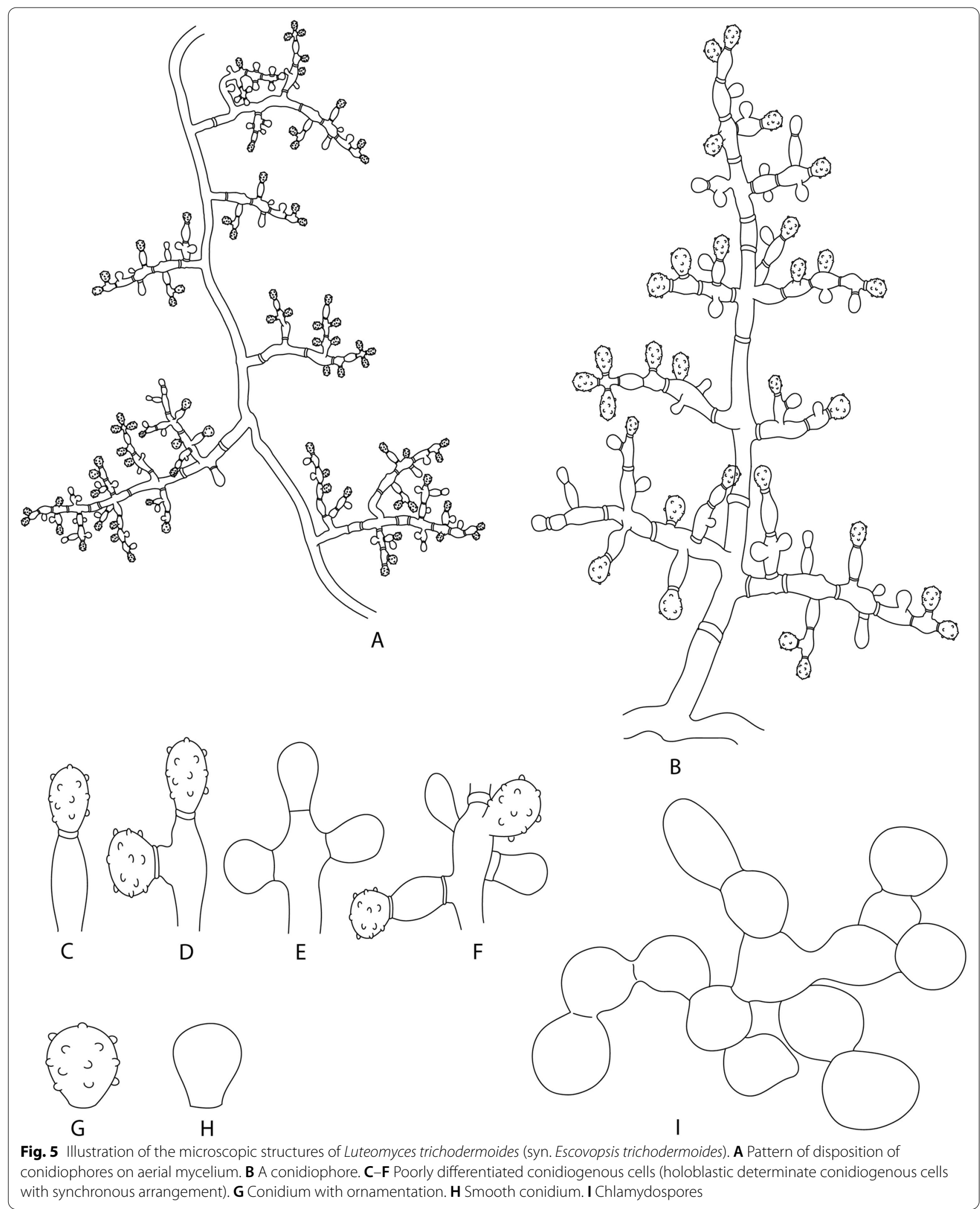




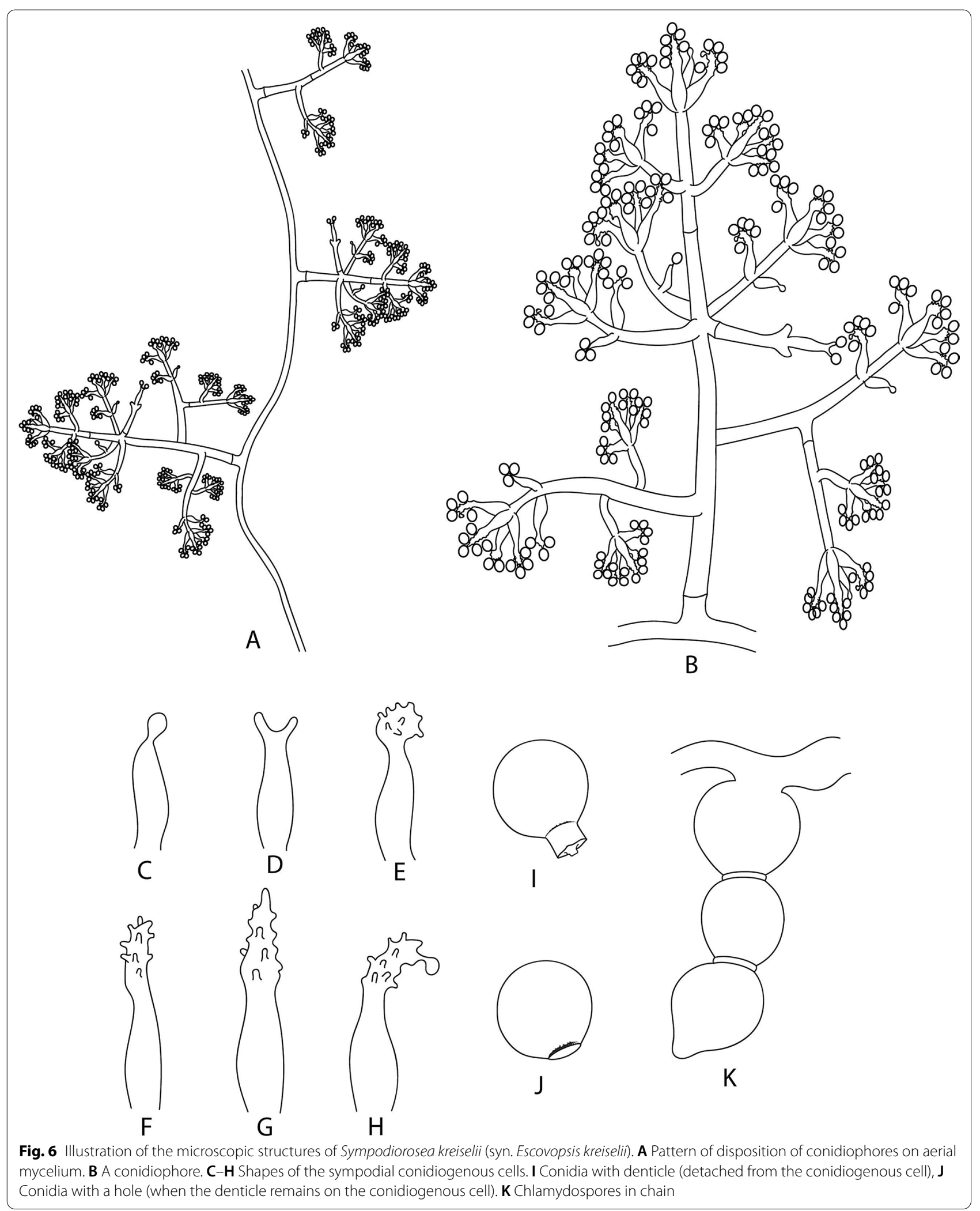


975 and LESF 979; LESF 969, LESF 996, LESF 997, and LESF 1003), which are all vesiculate species. We recognize these species as true Escovopsis because they form a monophyletic clade including the type species of the genus and preserve the main character (presence of vesicles on conidiophores) that gave origin to the generic name (Muchovej and Della Lucia 1990). Therefore, we redefine and restrict Escovopsis to include only vesiculate species (Fig. 3G) and the description of the genus is emended based on the criteria adopted in this study.

The two remaining clades (Fig. 3G) are made up of (1) E. kreiselii together with the three unnamed phylogenetic species, i.e., LESF 889, LESF 1010 and a group of strains (LESF 864, LESF 886 and LESF 887), and (2) E. trichodermoides (monotypic clade), which are only non-vesiculate Escovopsis species, respectively. Here, we establish two new genera, Sympodiorosea and Luteomyces, for reassigning these two non-vesiculate clades.

Escovopsis J.J. Muchovej \& Della Lucia, Mycotaxon 37: 192 (1990).

(Fig. 4).

MycoBank: MB 11249.

Type species: Escovopsis weberi J.J. Muchovej \& Della Lucia.

Original description: Muchovej and Della Lucia (1990), emended by Meirelles et al. (2015a).

Description: Monophyletic genus belonging to the Hypocreaceae that presents mono- to poly-vesiculate conidiophores formed on hyaline aerial mycelia (Fig. 4A-H). Vesicles terminal, mostly non-septate (Fig. 4I-T), rarely with one septum (Fig. 4U, V), various shaphed (globose, subglobose, capitate, obovoid, prolate, spatulate, clavate, cymbiform, lanceolate, subulate, cylindric, filiform, clavate-septate, cylindric-septate (Fig. 4I-V). Conidiogenous cells phialides, hyaline, with a thin base, a swollen section and a thin neck (Fig. 4W-Z), formed on vesicles. Conidia smooth or ornamented, aseptate, hyaline to brown, various shaped (globose, subglobose, ellipsoidal, cylindric; Fig. 4a-e), produced in chains.

Notes: Escovopsis is phylogenetically placed within the Hypocreaceae as a sister clade of Luteomyces (Fig. 3G). Escovopsis exhibits faster growth and different colony colour (brown) than Luteomyces (yellow). Unlike Luteomyces, which presents poorly diferenciated conidiogenous cells (i.e., holoblastic determinate conidiogenous cells with synchronous arrangement), Escovopsis forms phialides (i.e., enteroblastic conidiogenous cells). The main feature of this genus is the presence of conidiophores with terminal vesicles that differentiate it from its sister clade and from all other known genera in the Hypocreaceae.

\section{Luteomyces Q.V. Montoya \& A. Rodrigues, gen. nov.}

(Fig. 5).

\section{MycoBank: MB 835150.}

Etymology: "Luteomyces" based on the colour exhibited by the colonies of the type species.

Diagnosis: Similar to Escovopsis and Sympodiorosea in the way it begins to grow, i.e., dense germination and forming stolon-like mycelia. However, Luteomyces differs from these genera and other known genera in the Hypocreaceae by its poorly differentiated holoblastic conidiogenous cells.

Type species: Luteomyces trichodermoides (M. Cabello et al.) Q.V. Montoya \& A. Rodrigues 2021.

Description: Monophyletic genus in the Hypocreaceae. Colonies form floccose, white, beige and yellow mycelia, stolons, beige to yellow soluble pigments. Conidiophores formed on aerial mycelia, alternated, usually arising at right angles (Fig. 5A), smooth-walled, pyramidal shape (Fig. 5B). Conidiogenous cells poorly differenciated, holoblastic, determinate, in synchronous arrangement (Fig. 5C-F) on the apices and axes of conidiophores and their branches, solitary, ampulliform to lageniform (Fig. 5B). Conidia solitary, dry, smooth or ornamented (Fig. 5G, H), yellow to light-brown. Chlamydospores abundant, hyaline, smooth (Fig. 5I).

Notes: Luteomyces is phylogenetically placed in the Hypocreaceae as a sister clade of Escovopsis (Fig. 3G). Nonetheless, Luteomyces grows slower and has different colony colour (mainly yellow) than Escovopsis (mainly brown), and forms conidiophores without vesicles and large number of chlamydospores (rarely observed in Escovopsis).

Luteomyces trichodermoides (M. Cabello et al.) Q.V. Montoya \& A. Rodrigues, comb. nov.

(Fig. 5).

MycoBank: MB 835152. 
Basionym: Escovopsis trichodermoides M. Cabello et al., Antonie van Leeuwenhoek 107: 737 (2015).

Type: BRAZIL: Rio Claro, São Paulo, $22^{\circ} 23^{\prime} 46.93^{\prime \prime}$ S; $47^{\circ}$ $32^{\prime} 40.12^{\prime \prime} \mathrm{W}$, isolated from the upper part of a fungus garden of Mycocepurus goeldii, 13 Aug 2011. V. E. Masiulionis (14.0662-CBS holotype, preserved as a freezedried sample; VEM001, CBS 137343, CBMAI 1620, LPSC 1176-ex-type cultures).

Sequences: KJ485699 (ITS), KF033128 (tef1), MF116052 (LSU), MT305417 (rpb1), and MT305542 (rpb2).

Sympodiorosea Q.V. Montoya \& A. Rodrigues, gen. nov.

(Fig. 6).

MycoBank: MB 835147.

Etymology: "Sympodio" refers to the sympodial conidiogenous cells, and "rosea" to the colony colour of the type species.

Diagnosis: Similar to Escovopsis and Luteomyces in the way it begins to grow, i.e., dense germination and forming stolon-like mycelia. However, Sympodiorosea differs from these genera and other known genera in the Hypocreaceae by its holoblastic sympodial proliferous conidiogenous cells.

Type species: Sympodiorosea kreiselii (L.A. Meirelles et al.) Q.V. Montoya \& A. Rodrigues 2021.

Description: Monophyletic genus in the Hypocreaceae. Colonies form inconspicuous to floccose, white, palebeige, pink, brown aerial mycelia. Conidiophores formed on aerial mycelia (Fig. 6A), alternate or opposite, usually at right angles, with irregular branching conformation (Fig. 6A, B). Conidiogenous cells holoblastic, sympodial, proliferous (Fig. $6 \mathrm{C}-\mathrm{H}$ ), in pairs or in verticils on the apices of conidiophores and their branches, and solitary, alternate or opposite, on both the axes of the conidiophore and their branches (Fig. 6B). Conidia formed solitary, globose to subglobose, smooth or rough (thickwalled), light-brown to dark-brown, with denticles or lesions like holes (Fig. 6I, J). Chlamydospores commonly formed (Fig. 6K).

Notes: Sympodiorosea is phylogenetically placed in the Hypocreaceae near the genera Luteomyces and Escovopsioides (Fig. 3G). However, Sympodiorosea grows slower and has different colony colour (pink) than Luteomyces (yellow) and Escovopsioides (white). Sympodiorosea forms more branched conidiophores than Luteomyces and Escovopsioides.

Sympodiorosea kreiselii (L.A. Meirelles et al.) Q.V. Montoya \& A. Rodrigues, comb. nov.

(Fig. 6).

MycoBank: MB 835148.

Basionym: Escovopsis kreiselii L.A. Meirelles et al., PLoS One 10 (e0112067): 7 (2015).

Type: BRAZIL: Santa Catarina, Florianópolis, Praia da Joaquina, $27^{\circ} 37^{\prime} 50.01^{\prime \prime} \mathrm{S}$; $48^{\circ} 27^{\prime} 3.64^{\prime \prime} \mathrm{W}$, elev. $1 \mathrm{~m}$, isolated from fungus garden of Mycetophylax morschi, 6 Mar 2009. A. Rodrigues (CBS H-22062, dried culture on PDA - holotype; LESF 53, CBS 139320, CBMAI 1691ex-type cultures).

Sequences: KJ808767 (ITS), KJ808766 (tef1), KJ808765 (LSU), MT305418 (rpb1), and MT305543 (rpb2).

\section{Discussion}

Here, we provide the basis for the systematics of Escovopsis and related genera using a set of morphological characters, and comprehensive multilocus phylogenetic analyses. Our results supported the separation of species previously treated as Escovopsis into three distinct genera. Accordingly, we redefine and restrict Escovopsis to vesiculate species, and we describe Sympodiorosea to accommodate E. kreiselii and Luteomyces to accomodate E. trichodermoides. This study provides a long-awaited revision of Escovopsis systematics and related genera, thus helping future researchers to assess the diversity and evolutionary history of these fungus-growing ant associates.

Genera of the Hypocreaceae have morphological features that differentiate them from one another (Jaklitsch et al. 2008; Jaklitsch 2009; Põldmaa 2011). Due to the morphological plasticity of fungi (Slepecky and Starmer 2009; Wrzosek et al. 2017), variations in the shades of colours expressed by these organisms are highly expected. Nonetheless, the prevalent brown colour of Escovopsis colonies is a unique feature of this genus within the family. Curiously, other genera in the same family also exhibit unique colours, as is the case for the genus Trichoderma, which is characterized by its mostly green colonies (Jaklitsch 2009), and Escovopsioides, which is characterized by its white colour (Augustin et al. 2013). While Sympodiorosea and Luteomyces are currently monotypic genera, phylogenetic analysis suggests there are more Sympodiorosea species waiting to be described (Figs. 2, 
3G). Colours exhibited by these genera (pink and yellow, respectively) are also unique within the Hypocreaceae. Interestingly, the separation of these clades by the colour patterns was previously observed by other authors (Gerardo et al. 2006b; Meirelles et al. 2015b), but the lack of a deep morphological analysis prevented reaching the conclusion that they were different genera.

Microscopic features also differentiate Escovopsis from other genera in the Hypocreaceae. Conidiophores with terminal-vesicles producing phialides, present in Escovopsis, are a unique feature of the genus within this family. Escovopsioides also presents vesicles, however they are formed intercalary on aerial mycelia and solitary on the apex of the conidiophores. Furthermore, the vesicles of this genus are smaller and have fewer phialides than those of Escovopsis (Augustin et al. 2013). On the other hand, Sympodiorosea and Luteomyces are the only genera within the Hypocreaceae that present sympodial and poorly differentiated holoblastic conidiogenous cells, respectively. Interestingly, only a distant group of entomopathogenic fungi, i.e., Beauveria (Ascomycota: Hypocreales, Cordycipitaceae), has sympodial conidiogenesis (Rehner et al. 2011) like Sympodiorosea, and there are no other groups of fungi within the Hypocreaceae that form poorly differentiated holoblastic conidiogenous cells like Luteomyces. Future studies will hopefully shed light on the evolutionary pressures that led Escovopsis, Sympodiorosea, and Luteomyces to form these unique microscopic characters.

The consideration of all fungi producing brown conidia in the attine ant's colonies as Escovopsis made of this genus polyphyletic and paraphyletic (Fig. 1, Additional file 3: Fig. S2). Lack of a comprehensive phylogenetic analysis has precluded resolving the phylogenetic uncertainties of Escovopsis (Montoya et al. 2019). In light of our results, we considered two hypotheses to solve the phylogenetic disagreements of this group of fungi: first, Escovopsis, Luteomyces, and Sympodiorosea belong to the same genus. In this case, Escovopsioides (sister clade of Luteomyces, Fig. 3A, F) and Hypomyces (closely related with Sympodiorosea and Escovopsioides, Fig. 3C, D) would have to belong to the same genus to enforce monophyly (Baum and Smith, 2013). However, both Escovopsioides and Hypomyces are well supported, separate monophyletic clades (Figs. 1, 2, 3) and present unique morphological characters that differentiate them from other genera in the Hypocreaceae. Escovopsioides, for instance, is the only genus within the Hypocreaceae that forms phialides on tiny intercalary vesicles on the aerial mycelium. In contrast, many species of Hypomyces forms septate conidia and some species form sexual structures (despite being a physiological or genetic character not easily observed within the group), which are not observed in Escovopsis, Luteomyces, Sympodiorosea or Escovopsioides. Second, Escovopsis, Luteomyces, and Sympodiorosea represent taxa within different genera. Moreover, while different, Escovopsioides and Hypomyces (some species) are the only genera within the Hypocreaceae that form plural types of conidia (i.e., one type of conidia from phialides and another direct from aerial mycelia without conidiogenous cells (Põldmaa et al. 1999; Augustin et al. 2013). In this case, considering that: (1) the same genes, in different genera, follow different evolutionary paths (Gompel and Prud'homme 2009), and (2) regardless of the molecular markers used in this study, each clade preserves its monophyly within the Hypocreaceae; the variation of the phylogenetic position of the three clades (Fig. 3) could be better explained if they are different genera. In light of this evidence, the combination of morphological and phylogenetic data, using various molecular markers in a multilocus analysis (Taylor et al. 2000) suggests the second hypothesis as the most parsimonious. Future research, using genome-based phylogenetic methods, may resolve the relationship of these genera to one another.

The circumscription of Escovopsis raises important questions for the genus. How diverse is Escovopsis? What is its host range? How is the genus phylogeographically distributed? And, what is its role in attine gardens? For many years, Escovopsis was considered a diverse group of fungi (Gerardo et al. 2006a, b; Rodrigues et al. 2008, 2011; Caldera et al. 2009; Pagnocca et al. 2012; Yek et al. 2012; Meirelles et al. 2015a, b). However, that assumption was based on considering Sympodiorosea spp., L. trichodermoides and the clades $\mathrm{C}$ and $\mathrm{D}$ (putative new genera; Fig. 1, Additional file 3: Fig. S2) within Escovopsis. Currently, E. aspergilloides, E. clavata, E. lentecrescens, E. microspora, E. moelleri, E. multiformis, and E. weberi are the only species formaly described within the genus Escovopsis analysed using a multilocus approach based on five molecular markers (Fig. 3G). The species introduced by Marfetán et al. (2018) also belong to Escovopsis (Additional file 2: Fig. S1), nonetheless, the phylogeny of these species are still unclear. Therefore, future studies should consider that both the genetic diversity and the number of known species of the genus were overestimated. Since the taxonomic conditions to evaluate the macroscopic features and growth rates of Escovopsis species are still not standardized, the assessment to the morphological diversity of the genus and description of new species are still limited. Accordingly, future studies should access the morphology of the formally described Escovopsis species to create a standardized taxonomic framework of the genus, and strengthen the foundations of its systematics. Finally, several studies provided evidence that some Escovopsis species act as specialized 
mycoparasite (Currie et al. 1999a, 2003; Currie 2001; Gerardo et al. 2004; Little and Currie 2007; de Man et al. 2016). However, a recent study showed that Escovopsis species may act as an opportunistic fungus in attine ant colonies depending on host susceptibility (JiménezGómez et al. 2021). Either way, the parasitic mechanisms of the Escovopsis species that have a parasitic behaviour are still poorly understood, and the hypothesis of specialized mycoparasite for the genus was also raised considering Sympodiorosea spp., L. trichodermoides and the clades C and D (Fig. 1 and Additional file 3: Fig. S2) within the same genus. Therefore, future studies should unveil the phylogenetic correspondence of Escovopsis with the ants and the mutualistic fungi, and carefully address the mechanisms of the parasitism of this group of fungi.

Similar questions as those raised for Escovopsis must be addressed in future studies for Sympodiorosea, Luteomyces and the clades $\mathrm{C}$ and $\mathrm{D}$. The genetic and morphological diversity, as well as the geographical distribution of Sympodiorosea, Luteomyces and the two putative new genera (Fig. 1 and Additional file 3: Fig. S2), are still a mystery. Some authors suggested that Sympodiorosea spp. and L. trichodermoides are more likely to be associated with the colonies of lower attine ants than higher attine (e.g., leaf-cutting) ants (Gerardo et al. 2004, 2006b), but more evidence is necessary to confirm this hypothesis. Recent studies have shown that some strains of S. kreiselii behave as antagonists of the mutualistic fungus of Mycetophylax morschi being able to kill it under laboratory conditions (Custodio and Rodrigues 2019). However, the mechanisms by which it manages to kill the cultivars are completely unknown. On the other hand, Bizarria et al. (2020) demonstrated that L. trichodermoides had little negative impact on the mutualistic fungus of $\mathrm{Myco-}$ cepurus goeldii, being just able to inhibit the fungus cultivars in-vitro and unable to overcome defenses of the ant colonies. The taxonomy, ecology, and lifestyle of the clades C and D (Fig. 1 and Additional file 3: Fig. S2) previously treated as Escovopsis are still unknown. Therefore, all assumptions about these groups of fungi should be reconsidered and examined carefully in future studies.

\section{Conclusion}

Since Möller (1893) observed the "fungi of the strong conidial shapes" within the fungus gardens of fungusgrowing ants, several groups of fungi that share the same habitat were classified as Escovopsis. Many phylogenetic incongruities have been reported in the last two decades, and the lack of phylogenetic studies for this genus has hampered scientists to recognize the root of the problem. After a detailed systematic study, we conclude that taxonomic disagreements in the genus Escovopsis were caused due to the inclusion of two groups of fungi that belong to different genera (Luteomyces and Sympodiorosea) within the same genus. This discovery not only solves the phylogenetic disagreements of the genus but significantly expands our understanding of the systematics of Escovopsis, and related genera, and provides a stable foundation from which to build future research on the evolutionary history, taxonomic diversity, and ecological roles of these unique fungi.

\section{Supplementary Information}

The online version contains supplementary material available at https://doi. org/10.1186/s43008-021-00078-8.

Additional file 1. Table S1. Strains and their associated metadata used in the phylogenetic analyses at family level (Fig. 3). From these, 64 strains are from Escovopsis spp., 24 strains are from Luteomyces trichodermoides, 14 strains are from Sympodiorosea spp., 30 strains are from five Hypocreaceae genera [Escovopsioides, Hypomyces (along with species under its anamorphic genus (ladobotryum), Protocrea, Sphaerostilbella, and Trichoderma], and Lecanicillium antillanum CBS 350.85 was used as the outgroup. Table S2. Molecular markers, primers and polymerase chain reaction conditions used in this study. Table S3. Sequences and their associated metadata used to show the phylogenetic placement of all strains previously named as Escovopsis (Figs. 1 and S2). This table contains a total of 440 tef 1 sequences from 274 strains from vesiculate-Escovopsis spp., 105 strains from non-vesiculate Escovopsis [24 strains from Luteomyces trichodermoides (previously introduced as "Escovopsis trichodermoides"), 57 strains from Sympodiorosea spp. (9 Sympodiorosea kreiselii previously introduced as "Escovopsis kreiselii", 5 Sympodiorosea spp. introduced in this study, 6 Sympodiorosea spp. previously introduced as "Escovopsis", 37 Sympodiorosea spp. previously introduced as "Pink Escovopsis"), 24 strains labeled as "?" (because they form two new clades that likely correspond to new genera 20 previously introduced as "White Escovopsis", 2 as "Yellow Escovopsis", and 2 as "Escovopsis")], 60 strains from five Hypocreaceae genera [Escovopsioides, Hypomyces (along with species under its anamorphic genus Cladobotryum), Protocrea, Sphaerostilbella, and Trichoderma], and Lecanicillium antillanum CBS 350.85 as the outgroup. Table S4. Strains and their associated metadata used to show the phylogenetic placement of Escovopsis species described by Marfetán et al. (2018) (Fig. S1). Table S5. Strains of the Hypocreales and their metadata used in the phylogenetic analysis at order-level (Fig. 2).

Additional file 2: Figure S1. Phylogenetic placement of Escovopsis species described by Marfetán et al. (2018). The phylogenetic tree was reconstructed to include the LSU sequences (in the green box on the tree) generated by Marfetán et al. (2018). The tree was performed using Maximum Likelihood (ML) in RAxML v.8 (Stamatakis 2014) and Bayesian Inference (BI) in MrBayes v.3.2.2 (Ronquist et al. 2012) using the GTR model. For ML analyses, 1000 independent trees and 1000 bootstrap replicates were generated. For Bl analyses, two million generations of the Markov Chain Monte Carlo were enough to reach convergence. Numbers on branches indicate $\mathrm{Bl}$ posterior probabilities (PP) and ML bootstrap support values (MLB), respectively. Hyphens (--) indicate MLB $<70 \%$. Lecanicillium antillanum CBS 350.85 was used as the outgroup. Four species described by Marfetán et al. (2018) (Escovopsis atlas, E. catenulata, E. pseudoweberi, and E. primorosea) formed two clades (green box) within Escovopsis close to E. aspergilloides and E. lentecrescens. Escovopsis atlas, E. catenulata, E. pseudoweberi were placed into the same clade along with five strains identified as E. weberi (distant from the type of E. weberi) and four strains of E. primorosea formed a monophyletic clade. Escovopsis longivesica was not included in this tree because the LSU sequences of this species do not have similarity with Escovopsis but with Ceriporia alachuana (95.4\% identity for E. longivesica E5 and E. Iongivesica E9) and Penicillium glabrum (95.3\% identity for E. longivesica E10). See Additional file 1: Table S4 for all strains and their associated metadata used to infer this phylogenetic tree. 
Additional file 3: Figure S2. Extended phylogenetic tree (from Fig. 1) indicating the placement of every isolate previously treated as Escovopsis. The tree shown was inferred using Bayesian Inference (BI). The tree gathers all available tef1 sequences found in the literature and the data set used in this study, including the sequences of the nine Escovopsis ex-type cultures. The tree contains a total of 440 sequences which include: 274 strains of vesiculate-Escovopsis (Clade A), 105 strains of non-vesiculate Escovopsis (Clades B, C, D, E) and 60 strains from four genera, i.e., Escovopsioides, Hypomyces (along with species under its anamorphic genus Cladobotryum), Protocrea, and Trichoderma, in the Hypocreaceae. Lecanicillium antillanum CBS 350.85 was used as the outgroup. Numbers on branches indicate BI posterior probabilities (PP) and Maximum Likelihood bootstrap support values (MLB), respectively. Hyphens (--) indicate MLB $<70 \%$. There is only information, in the literature, on the colour of the colonies of the clades $C$ and $D$, but the microscopic features of these clades are unknown. See Additional file 1: Table S3 for all strains and their associated metadata used to infer this phylogenetic tree.

\section{Acknowledgements}

We would like to thank the Fungal Ecology and Systematics (LESF_-São Paulo State University, Rio Claro, SP, Brazil) and Gerardo Laboratory (Emory University, Atlanta, USA) research teams, especially Dr. Aileen Berasategui Lopez, Dr. Caitlin Conn, Dr. Pepijn Kooij and MSc. Mariana Barcoto, for valuable comments on this manuscript. We would like to thank the associate editor and the three anonymous reviewers for valuable comments on this manuscript. We would like to thank the Biological Dynamics of Forest Fragmentation Project (BDFFP) for providing logistical and field support. This is publication number 820 of the Technical Series of the BDFFP (INPA/STRI).

\section{Authors' contributions}

QVM and AR designed the study. QVM carried out the morphological and phylogenetic analyses. QVM, MJSM, RBJ carried out in vitro growth experiments. QVM, AR, MSJM and NMG wrote the manuscript. All authors read and approved the final manuscript.

\section{Funding}

We are grateful to Fundação de Amparo à Pesquisa do Estado de São Paulo (FAPESP) for financial support (Grants \# 2014/24298-1, \# 2017/12689-4 and \#2019/03746-0) of AR and for scholarships (\# 2016/04955-3 and \# 2018/079313) to QVM. We are also grateful to the National Science Foundation for financial support of NMG (NSF DEB-1754595 and DEB-1927161). AR also thanks Conselho Nacional de Desenvolvimento Científico e Tecnológico (CNPq) for a fellowship (Grant \# 305269/2018-6).

\section{Availability of data and materials}

The datasets used in this study are available in the NCBI-GenBank (Additional file 1:Tables S1, S3, S4, and S5). The alignments and their respective phylogenetic trees generated in this study are available in TreeBASE (http://purl.org/ phylo/treebase/phylows/study/TB2:S28662).

\section{Adherence to national and international regulations}

We followed all the requirements for specimen acquisition, transportation and study according to the Nagoya Protocol to the Convention on Biological Diversity 2011.

\section{Declarations}

Ethics approval and consent to participate

Not applicable.

\section{Consent for publication}

Not applicable.

\section{Competing interests}

The authors declare that they have no competing interests.

\section{Author details}

${ }^{1}$ Department of General and Applied Biology, São Paulo State University (UNESP), Avenida 24-A, n. 1515, Bela Vista, Rio Claro, SP 13.506-900, Brazil. ${ }^{2}$ Center for the Study of Social Insects, São Paulo State University (UNESP), Rio Claro, SP, Brazil. ${ }^{3}$ Department of Biology, O. Wayne Rollins Research Center, Emory University, Atlanta, USA.

Received: 11 November 2020 Accepted: 10 August 2021

Published online: 24 August 2021

\section{References}

Augustin JO, Groenewald JZ, Nascimento RJ, Mizubuti ESG, Barreto RW, Elliot SL, Evans HC (2013) Yet more "weeds" in the garden: fungal novelties from nests of leaf-cutting ants. PLoS ONE 8:e82265. https://doi.org/10.1371/ journal.pone.0082265

Baum DA, Smith SD (2013) Tree thinking: an introduction to phylogenetic biology. Macmillan Learning. New York. ISBN: 9781936221165

Birnbaum SSL, Gerardo NM (2016) Patterns of specificity of the pathogen Escovopsis across the fungus-growing ant symbiosis. Am Nat 188:52-65. https://doi.org/10.1086/686911

Bizarria R, Nagamoto NS, Rodrigues A (2020) Lack of fungal cultivar fidelity and low virulence of Escovopsis trichodermoides. Fungal Ecol 45:100944. https://doi.org/10.1016/j.funeco.2020.100944

Caldera EJ, Poulsen M, Suen G, Currie CR (2009) Insect symbioses: a case study of past, present, and future fungus-growing ant research. Environ Entomol 38:78-92. https://doi.org/10.1603/022.038.0110

Castellani A (1963) Further researches on the long viability and growth of many pathogenic fungi and some bacteria in sterile distilled water. Mycopathol Mycol Appl 20:1-6. https://doi.org/10.1007/BF02054872

Currie CR (2001) Prevalence and impact of a virulent parasite on a tripartite mutualism. Oecologia 128:99-106. https://doi.org/10.1007/s004420100 630

Currie CR, Mueller UG, Malloch D (1999a) The agricultural pathology of ant fungus gardens. Proc Natl Acad Sci USA 96:7998-8002. https://doi.org/10. 1073/pnas.96.14.7998

Currie CR, Scott JA, Summerbell RC, Malloch D (1999b) Fungus-growing ants use antibiotic-producing bacteria to control garden parasites. Nature 398:701-704. https://doi.org/10.1038/nature01563

Currie CR, Wong B, Stuart AE, Schultz TR, Rehner SA, Mueller UG, Sung GH, Spatafora JW, Straus NA (2003) Ancient tripartite coevolution in the attine ant-microbe symbiosis. Science 299:386-388. https://doi.org/10.1126/ science. 1078155

Custodio BC, Rodrigues A (2019) Escovopsis kreiselii specialization to its native hosts in the fungiculture of the lower attine ant Mycetophylax morschi. Antonie Van Leeuwenhoek 112:305-317. https://doi.org/10.1007/ s10482-018-1158-x

Darriba D, Taboada GL, Doallo R, Posada D (2012) jModelTest 2: more models, new heuristics and parallel computing. Nat Methods 9:772-772. https:// doi.org/10.1038/nmeth.2109

de Man TJB, Stajich JE, Kubicek CP, Teiling C, Chenthamara K, Atanasova L, Druzhinina I, Levenkova N, Birnbaum SSL, Barribeau SM, Bozick BA, Suen G, Currie CR, Gerardo NM (2016) Small genome of the fungus Escovopsis weberi, a specialized disease agent of ant agriculture. Proc Natl Acad Sci USA 113:3567-3572. https://doi.org/10.1073/pnas.1518501113

Elizondo Wallace DE, Vargas Asensio JG, Pinto Tomás AA (2014) Correlation between virulence and genetic structure of Escovopsis strains from leafcutting ant colonies in Costa Rica. Microbiology 160:1727-1736. https:// doi.org/10.1099/mic.0.073593-0

Folgarait P, Gorosito N, Poulsen M, Currie CR (2011) Preliminary in vitro insights into the use of natural fungal pathogens of leaf-cutting ants as biocontrol agents. Curr Microbiol 63:250-258. https://doi.org/10.1007/ s00284-011-9944-y

Gerardo NM, Mueller UG, Price SL, Currie CR (2004) Exploiting a mutualism: parasite specialization on cultivars within the fungus-growing ant symbiosis. Proc R Soc B Biol Sci 271:1791-1798. https://doi.org/10.1098/ rspb.2004.2792

Gerardo NM, Jacobs SR, Currie CR, Mueller UG (2006a) Ancient host-pathogen associations maintained by specificity of chemotaxis and antibiosis. PLoS Biol 4:1358-1363. https://doi.org/10.1371/journal.pbio.0040235 
Gerardo NM, Mueller UG, Currie CR (2006b) Complex host-pathogen coevolution in the Apterostigma fungus-growing ant-microbe symbiosis. BMC Evol Biol 6:88. https://doi.org/10.1186/1471-2148-6-88

Gompel N, Prud'homme B (2009) The causes of repeated genetic evolution. Dev Biol 332:36-47. https://doi.org/10.1016/j.ydbio.2009.04.040

Hall T (1999) BioEdit: a user-friendly biological sequence alignment editor and analysis program for Windows 95/98/NT. Nucleic Acids Symp Ser 41:95-98

Hawksworth DL, Lücking R (2017) Fungal diversity revisited: 2.2 to 3.8 million species. Microbiol Spectr 1:1-17. https://doi.org/10.1128/microbiolspec. FUNK-0052-2016

Heine D, Holmes NA, Worsley SF, Santos ACA, Innocent TM, Scherlach K, Patrick EH, Yu DW, Murrell JC, Vieria PC, Boomsma JJ, Hertweck C, Hutchings MI, Wilkinson B (2018) Chemical warfare between leafcutter ant symbionts and a co-evolved pathogen. Nat Commun 9(2208):1-11. https://doi.org/ 10.1038/s41467-018-04520-1

Jaklitsch WM (2009) European species of Hypocrea part I. The green-spored species. Stud Mycol 63:1-91. https://doi.org/10.3114/sim.2009.63.01

Jaklitsch WM, Poldmaa K, Samuels GJ (2008) Reconsideration of Protocrea (Hypocreales, Hypocreaceae). Mycologia 100:962-984

Jiménez-Gómez I, Barcoto MO, Montoya QV, Goes AC, Monteiro LSVE, Bueno OC, Rodrigues A (2021) Host susceptibility modulates Escovopsis pathogenic potential in the fungiculture of higher attine ants. Front Microbiol 12:673444. https://doi.org/10.3389/fmicb.2021.673444

Katoh K, Standley DM (2013) MAFFT multiple sequence alignment software version 7: improvements in performance and usability. Mol Biol Evol 30:772-780. https://doi.org/10.1093/molbev/mst010

Kearse M, Moir R, Wilson A, Stones-Havas S, Cheung M, Sturrock S, Buxton S, Cooper A, Markowitz S, Duran C, Thierer T, Ashton B, Meintjes P, Drummond A (2012) Geneious basic: an integrated and extendable desktop software platform for the organization and analysis of sequence data. Bioinformatics 28:1647-1649. https://doi.org/10.1093/bioinformatics/ bts199

Komarek A, Beutel RG (2006) Problems in taxonomy and suggestions for a standardized description of new insect taxa. Entomol Probl 36:55-70

Kreisel H (1972) Pilze aus Pilzgärten von Atta insularis in Kuba. Z Allg Mikrobiol 12:643-654. https://doi.org/10.1002/jobm.19720120805

Little AEF, Currie CR (2007) Symbiotic complexity: discovery of a fifth symbiont in the attine ant-microbe symbiosis. Biol Lett 3:501-504. https://doi.org/ $10.1098 /$ rsbl.2007.0253

Marfetán JA, Romero Al, Folgarait PJ (2015) Pathogenic interaction between Escovopsis weberi and Leucoagaricus sp.: mechanisms involved and virulence levels. Fungal Ecol 17:52-61. https://doi.org/10.1016/j.funeco. 2015.04.002

Marfetán JA, Romero Al, Cafaro MJ, Folgarait PJ (2018) Five new Escovopsis species from Argentina. Mycotaxon 133:569-589. https://doi.org/10. $5248 / 133.569$

Masiulionis VE, Cabello MN, Seifert KA, Rodrigues A, Pagnocca FC (2015) Escovopsis trichodermoides sp. nov., isolated from a nest of the lower attine ant Mycocepurus goeldii. Antonie Van Leeuwenhoek 107:731-740. https://doi. org/10.1007/s10482-014-0367-1

Meirelles LA, Montoya QV, Solomon SE, Rodrigues A (2015a) New light on the systematics of fungi associated with attine ant gardens and the description of Escovopsis kreiselii sp. nov. PLoS ONE. https://doi.org/10.1371/journ al.pone.0112067

Meirelles LA, Solomon SE, Bacci M, Wright AM, Mueller UG, Rodrigues A (2015b) Shared Escovopsis parasites between leaf-cutting and non-leafcutting ants in the higher attine fungus-growing ant symbiosis. R Soc Open Sci 2:150257. https://doi.org/10.1098/rsos.150257

Möller AFW (1893) Die Pilzgärten einiger südamerikanischer Ameisen. Gustav Fischer, Jena

Möller EM, Bahnweg G, Sandermann H, Geiger HH (1992) A simple and efficient protocol for isolation of high molecular weight DNA from filamentous fungi, fruit bodies, and infected plant tissues. Nucleic Acids Res 20:6115-6116

Montoya QV, Martiarena MJS, Polezel DA, Akazu S, Rodrigues A (2019) More pieces to a huge puzzle: two new Escovopsis species from fungus gardens of attine ants. MycoKeys 46:97-118. https://doi.org/10.3897/mycok eys.46.30951
Muchovej JJ, Della Lucia TMC (1990) Escovopsis, a new genus from leafcutting ants nests to replace Phailocladus nomem invalidum. Mycotaxon 37:191-195

Mueller GM, Schmit JP (2007) Fungal biodiversity: what do we know? What can we predict? Biodivers Conserv 16:1-5. https://doi.org/10.1007/ s10531-006-9117-7

Nixon KC (2002) WinClada ver. 1.0000. Publ by author, Ithaca

Pagnocca FC, Masiulionis VE, Rodrigues A (2012) Specialized fungal parasites and opportunistic fungi in gardens of attine ants. Psyche. https://doi.org/ 10.1155/2012/905109

Pavlinov IY (2018) Foundations of biological systematics: history and theory. KMK Scientific Press, Moscow

Põldmaa K (2011) Tropical species of Cladobotryum and Hypomyces producing red pigments. Stud Mycol 68:1-34. https://doi.org/10.3114/sim.2011.68. 01

Pöldmaa K, Larsson E, Kõljalg U (1999) Phylogenetic relationships in Hypomyces and allied genera, with emphasis on species growing on wooddecaying homobasidiomycetes. Can J Bot 77:1756-1768. https://doi.org/ 10.1139/b99-148

Rehner SA, Minnis AM, Sung GH, Luangsa-ard JJ, Devotto L, Humber RA (2011) Phylogeny and systematics of the anamorphic, entomopathogenic genus Beauveria. Mycologia 103:1055-1073. https://doi.org/10.3852/ 10-302

Rodrigues A, Bacci M, Mueller UG, Ortiz A, Pagnocca FC (2008) Microfungal "weeds" in the leafcutter ant symbiosis. Microb Ecol 56:604-614. https:// doi.org/10.1007/s00248-008-9380-0

Rodrigues A, Mueller UG, Ishak HD, Bacci M, Pagnocca FC (2011) Ecology of microfungal communities in gardens of fungus-growing ants (Hymenoptera: Formicidae): a year-long survey of three species of attine ants in Central Texas. FEMS Microbiol Ecol 78:244-255. https://doi.org/10.1111/j. 1574-6941.2011.01152.x

Ronquist F, Teslenko M, van der Mark P, Ayres DL, Darling A, Höhna S, Larget B, Liu L, Suchard MA, Huelsenbeck JP (2012) MrBayes 3.2: efficient Bayesian phylogenetic inference and model choice across a large model space. Syst Biol 61:539-542. https://doi.org/10.1093/sysbio/sys029

Seifert KA, Samson RA, Chapela IH (1995) Escovopsis aspergilloides, a rediscovered hyphomycete from leaf-cutting ant nests. Mycologia 87:407-413

Slepecky RA, Starmer WT (2009) Phenotypic plasticity in fungi: a review with observations on Aureobasidium pullulans. Mycologia 101:823-832. https://doi.org/10.3852/08-197

Stamatakis A (2014) RAxML version 8: a tool for phylogenetic analysis and post-analysis of large phylogenies. Bioinformatics 30:1312-1313. https:// doi.org/10.1093/bioinformatics/btu033

Sung GH, Poinar GO, Spatafora JW (2008) The oldest fossil evidence of animal parasitism by fungi supports a Cretaceous diversification of fungalarthropod symbioses. Mol Phylogenet Evol 49:495-502. https://doi.org/ 10.1016/j.ympev.2008.08.028

Taerum SJ, Cafaro MJ, Little AE, Schultz TR, Currie CR (2007) Low host-pathogen specificity in the leaf-cutting ant-microbe symbiosis. Proc $R$ Soc $B$ Biol Sci 274:1971-1978. https://doi.org/10.1098/rspb.2007.0431

Taerum SJ, Cafaro MJ, Currie CR (2010) Presence of multiparasite infections within individual colonies of leaf-cutter ants. Environ Entomol 39:105-113. https://doi.org/10.1603/EN09137

Taylor JW, Jacobson DJ, Kroken S, Kasuga T, Geiser DM, Hibbett DS, Fisher MC (2000) Phylogenetic species recognition and species concepts in fungi. Fungal Genet Biol 31:21-32. https://doi.org/10.1006/fgbi.2000.1228

Wrzosek M, Ruszkiewicz-Michalska M, Sikora K, Damszel M, Sierota Z (2017) The plasticity of fungal interactions. Mycol Prog 16:101-108. https://doi. org/10.1007/s11557-016-1257-x

Yek SH, Boomsma JJ, Poulsen M (2012) Towards a better understanding of the evolution of specialized parasites of fungus-growing ant crops. Psyche 2012:239392. https://doi.org/10.1155/2012/239392

\section{Publisher's Note}

Springer Nature remains neutral with regard to jurisdictional claims in published maps and institutional affiliations. 